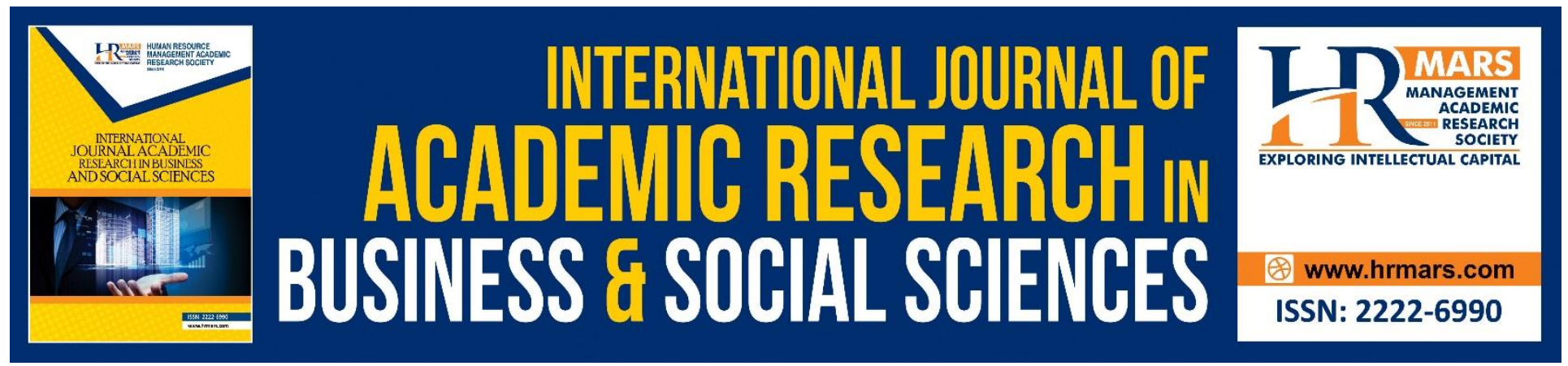

\title{
Issues and Challenges of Mental Health in Malaysia
}

\author{
Mohd Faizul bin Hassan, Naffisah Mohd Hassan, Erne Suzila Kassim and \\ Muhammad Iskandar Hamzah
}

To Link this Article: http://dx.doi.org/10.6007/IJARBSS/v8-i12/5288

DOI: $10.6007 /$ IJARBSS/v8-i12/5288

Received: 13 Nov 2018, Revised: 06 Dec 2018, Accepted: 30 Dec 2018

Published Online: 31 Dec 2018

In-Text Citation: (Hassan, Hassan, Kassim, \& Hamzah, 2018)

To Cite this Article: Hassan, M. F. bin, Hassan, N. M., Kassim, E. S., \& Hamzah, M. I. (2018). Issues and Challenges of Mental Health in Malaysia. International Journal of Academic Research in Business and Social Sciences, 8(12), 1685-1696.

Copyright: (C) 2018 The Author(s)

Published by Human Resource Management Academic Research Society (www.hrmars.com)

This article is published under the Creative Commons Attribution (CC BY 4.0) license. Anyone may reproduce, distribute, translate and create derivative works of this article (for both commercial and non-commercial purposes), subject to full attribution to the original publication and authors. The full terms of this license may be seen

at: http://creativecommons.org/licences/by/4.0/legalcode

Vol. 8, No. 12, 2018, Pg. 1685 - 1696

http://hrmars.com/index.php/pages/detail/IJARBSS

JOURNAL HOMEPAGE

Full Terms \& Conditions of access and use can be found at http://hrmars.com/index.php/pages/detail/publication-ethics 


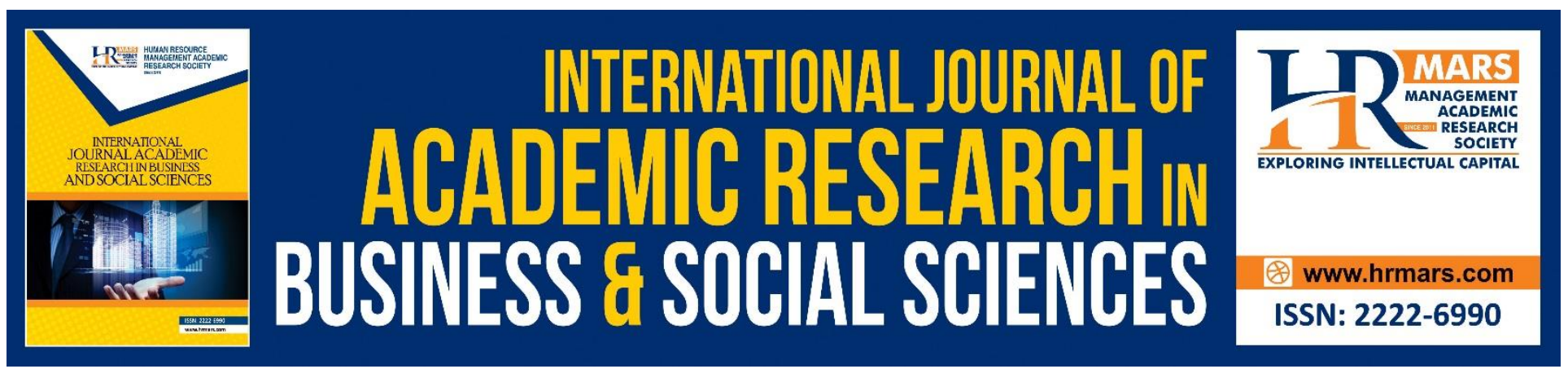

\title{
Issues and Challenges of Mental Health in Malaysia
}

\author{
${ }^{1}$ Mohd Faizul bin Hassan, ${ }^{1 *}$ Naffisah Mohd Hassan, ${ }^{1}$ Erne Suzila \\ Kassim and ${ }^{1}$ Muhammad Iskandar Hamzah \\ ${ }^{1}$ Faculty of Business and Management, Universiti Teknologi MARA Cawangan Selangor, \\ Kampus Puncak Alam, 42300, Selangor, MALAYSIA \\ Email: "naffi885@puncakalam.uitm.edu.my
}

\begin{abstract}
Mental health disorder is no crime. It needs treatment, not stigma it's a global alarming where the number of people who suffering with mental health illness keep increasing. Mental health problems are one of the main causes of the overall disease burden worldwide. Mental health illness is a major community health concern where depression and anxiety name as the two most common mental illness. Furthermore, depression is a leading cause of disability worldwide. Mental disorders represent a major contributor to disease burden worldwide it also affects to the economic burden. Most people have bad stigma towards this issue. Mental health conditions are treatable. Nevertheless, mental illness can affect anyone regardless of the age, income, social status, race ethnicity, religion/spirituality, background or anything others aspect of culture. The issues and challenge of mental health in Malaysia is explored as to give a big picture of the current situation that happen today. Therefore, it raises the question on how the mental health issues can solve as to reduce the statistic and improve their quality of life. In this paper, a case study was conducted to gather insights from community their understanding regarding the issues and challenges of mental health in Malaysia.
\end{abstract}

Keywords: Mental Health, Mental III, Global Burden

\section{INTRODUCTION}

Mental health disorder is a great public health concern throughout the world. It became an integral part of Sustainable Development Goals agenda to transform the world by 2030. It contributes to a substantial proportion of health problems in most countries Mental Health issues are expected to be major problems among Malaysians. Mental health problems are among the most important contributors to the burden of disease and disability worldwide. The global burden of mental health disorders is a significant of public health issues (Organization, 2017). Mental illness is a major source of loss of productivity and wellbeing as mental health is essential for economic development. Generally Mental health is the condition which is its influenced mind in dealing the daily activities. It 
also determined on how people handle the stress and how the individuals relate to others and how to make choices (mentalhealth.gov, 2017).

The World Health Organization (WHO) (Organization, 2013) defines the Mental Health as a state of wellbeing in which the individual realizes their own abilities to cope the level of stresses of life which is can be productively and fruitfully and ability to make contribution to their community from the Malaysia landscape the Ministry of Health (1997:2) has defined of the capacity of the Individual, the group and environment to interact with one another $r$ to promote subjective well-being and optimal functioning, and the use of cognitive, affective and relational abilities, towards the achievement of individual and collective goals consistent with justice"

In Malaysia, the National Health and Morbidity Survey, conducted by Ministry of Health (MOH), indicates that the prevalence of mental health problems among people aged 16 years and above was 29.2\% as approximately 4.2 million. From this figure it shows that one in three Malaysian has experienced the mental health problems. The current situation is very worrying as the findings indicates that twofold increase for the reported cases related to the mental health problem over the past 10 years of $10.6 \%$ in 1196 and $11.2 \%$ in 2006 . The sufferers of mental health problem may bring about devastating impact towards their self and tends to develop suicidal behavior. The proper assistance and guidance need to be provided to them. (Rotenstein et al., 2016) In other words, mental health problems are commonly higher among younger adults, with adolescents aged 16 to 19 (34.7\%), followed by those aged 20 to 24 (32.1\%), and those aged 25 to 29 (30.5\%) (Ahmad et al., 2015)

\section{LITERATURE REVIEW}

\section{Mental Health in Malaysia}

Malaysia's Ministry of Health (KKM), recently has been reported that one in three of the country's adults are at risk of developing a mental health problem for the last 5 years (Health, 2011). Mental illness is expected to be the second highest form of health problem after heart disease by 2020 (Lee \& Lai, 2017). Generally, mental health is related to the depression, anxiety and stress. By the year 2020, mental health issues are expected to be major problems among Malaysian. According to the National Health and Morbidity Survey 2015, it is indicated that mental illness is expected to be the second biggest health problem affecting Malaysians after heart diseases by 2020 and every 3 in 10 adults aged 16 years and above (29.2\%) have some sorts of mental health problems. The Health ministry statistics revealed of increasing number of mental health problems. The workplace is one of the key environments that affect our mental wellbeing and health. In other hand some research has been proved the mental health among students also keep increasing yearly (Zivin, Eisenberg, Gollust, \& Golberstein, 2009).

\section{Challenges on Mental Health}

There are many challenges towards on mental health in Malaysia especially Public Attitudes towards Mental illness (Yeap \& Low, 2009) indicates that $62.3 \%$ of the mental illness sufferers would not let others know about their conditions, meanwhile $61.0 \%$ believed sufferers are not to be blamed 
for their own conditions and $51.7 \%$ believed that people with mental illness are often dangerous and violent. Vast of majority $76.5 \%$ of respondents don't believe that anyone can suffer from mental health problems. Stigma and beliefs also to be the main factors that contributing of challenges as it is dominated by religious or spiritual explanation like demon possession. Malaysia comprises three main ethnic groups: Malays (61.7\%), Chinese (20.8\%) and Indians (6.2\%). These ethnic cultural has brought a different definition of mental health" that may different perspective on what the mental health really is.

As a matter of fact, the demands of living nowadays drive to the causing of depression, anxiety and other mental health problems. Unemployment, financial crisis, marital problems, drug abuse and surrounding factors that could have contributed to the twofold jump in mental cases in Malaysia over the last 10 years. (Chowdhury, Islam, \& Lee, 2013). Furthermore, the prevalence of mental health problem is highest among teenagers and low-income earners. The demands of living in current times are causing depression, anxiety and other mental health problems in young people.

From this study its revealed most of the respondents not familiar and well understand the term of mental health especially the definition of mental health illness.

Table 1. Definition of terminology of Mental Health. (Source: The National Institute of Mental Health (NIMH).

\begin{tabular}{ll}
\hline Type & Definition \\
\hline Schizophrenia & A severe mental disorder that appears in late adolescence \\
or early adulthood. People with schizophrenia may have \\
hallucinations, delusions, loss of personality, confusion, \\
agitation, social withdrawal, psychosis and/or extremely \\
odd behavior. \\
Manic disorder is characterized by extreme and \\
unpredictable mood changes in the sufferers \\
A disorder that causes severe and unusually high and low \\
shifts in mood, energy, and activity levels as well as \\
unusual shifts in the ability to carry out day-to-day tasks. \\
(Also known as Manic Depression) \\
Lack of interest or pleasure in daily activities, sadness and \\
feelings of worthlessness or excessive guilt that are severe \\
enough to interfere with working, sleeping, studying, \\
eating and enjoying life.
\end{tabular}

\section{RESEARCH METHODS}

\section{Research Design}

Since the aim of the research is to gain insights and capture the richness of the key issues and challenges, a case study via sets of interviews was conducted. The method is believed to provide empirical inquiries that investigate the contemporary phenomenon. Furthermore, the purpose and 
focus of the method is to describe the meaning, provide deep understanding, and interpretation of the textual information derived from the interviews. In order to gain a holistic comprehension and reflection, the interviews were conducted with the key stakeholders; health professionals, working adults who used to suffers with mental health disorders, and healthy adults. Participants that represent the three different groups were selected based on purposive sampling strategy.

Unstructured interviews were conducted that allows for probing the answers for more input. In addition, by using the interview technique, the respondents had the freedom to discuss the issues on a broader topic. Therefore, even though the aim of the study was to get insights of the key issues and challenges of mental health, we started by asking about the mental health in general. Once the respondents provided their feedback, we probe further by asking "why", "how" and "what".

\section{Respondents Background}

Unique purposeful sampling method was applied in this study. The research was interested to understand the issues and challenges of mental health from the viewpoint of the society. Therefore, purposeful sampling was appropriate assuming that researcher wants to discover, understand and gain insight about the topic; thus selecting sample (which will now then been referred to as "informant") from which can be learned is crucial.

The informants were selected based on unique sampling, that referred to selection of sampling based on their unique and atypical attributes, whom the researcher believes were able to provide honest answers that will relevantly answering the research questions and assisting in achieving research objectives. Through a personal contact, 10 informants ( 2 health professionals, 3 working adults who used to suffers with mental health disorders, and 5 healthy adults) had been approached and interviewed. They were chosen as the informants due to their profile that met the criterions set by the researchers.

\section{RESULTS AND DISCUSSION}

The data was analyzed by following the constant comparison method. This was highlighted by Boeije (2002) that the aim of the constant comparison method is to discover the concepts. There were four phases involved namely, exploration, specification, reduction and integration. Following Corbin and Strauss (2014) three coding processes of open, axial and selective were adhered to in the exploration phase. The validation of the findings was achieved by getting the participants' approval on the narrative summary. The findings suggest for the following challenges and issues: lack of awareness and ignorance, stigma, policy insurance, rising trend of mental illness, work-related stress and negative economic impact. The summary of the findings is presented in the figure below. 
INTERNATIONAL JOURNAL OF ACADEMIC RESEARCH IN BUSINESS AND SOCIAL SCIENCES

Vol. 8, No. 12, Dec, 2018, E-ISSN: 2222-6990 @ 2018 HRMARS

The summary of the findings is presented in the figure below.

Fig 1. Summary of key findings

\begin{tabular}{lll}
\hline No & Theme & Sub-theme \\
\hline 1 & Challenges & 1.1 Lack of Awareness and Ignorance \\
& & 1.2 Stigma \\
& 1.3 Policy Insurance \\
\hline 2 & Issues & 2.1 Rising trend of Mental IIIness \\
& 2.2 Work-related Stress \\
& 2.3 Negative Economic Impact \\
\hline
\end{tabular}

Detail discussion of the key findings are presented here

\section{Challenges}

The issues of mental health landscape have been spotlighted as critical issues due the number of mental illness keep increasing year by year. there is an urgent need to further enlighten by government and society to take this as serious matters as it give impact to the economic burden as stated by (Teoh et al., 2017). The economic burden associated with Schizophrenia (SCZ) as substantial and mainly attributed to productivity loss

\section{Lack of Awareness}

Many of the respondents believe that the issue of mental illness is something that is not being given enough exposure and attention in the mass media space. Some of them pointed out that it might be due to complacent behaviour of the mental health sufferers. These people might have experienced certain levels of cognitive dissonance within themselves, in the sense that they would not admit themselves suffering from mental illness despite the obvious symptoms.

Lack of awareness about the mental illnesses poses a challenge to the mental health issues. Mental illness literacy is the knowledge and belief that recognized mental illness problems. A research by (Kaur et al., 2014) revealed that almost one in five Malaysian adolescents are depressed. One reason for this condition is because the insufficiency of knowledge about psychiatric illness and treatment options for those illnesses (Loo \& Furnham, 2012). (Brown, Harris, \& Russell, 2010) With the proper mental health awareness campaign, it can be promising the positive outcomes which is can enhance the mental health literacy which is increasingly the awareness and knowledge of the mental health disorders. Generally, the definition of health literacy can been described as "ability to access, understand to use the information as to promote and maintain good health (Bull, Schipper, Jamrozik, \& Blanksby, 1997). By having mental health literacy, it believes that people have basic knowledge when they suffer or facing the symptoms. They will attempt to manage those symptoms.

Globally, it is stated that more than $70 \%$ of people with mental illness did not receive treatment from health care staff. (Henderson, Evans-Lacko, \& Thornicroft, 2013). The public needs to be equipped with sufficient knowledge about mental illness it believes it can ensure the people with minor or major symptoms will get a proper treatment that they need. Some of the respondents highlighted the conservative attitude of family members of mental health patients, in the sense that they are 
hesitant in seeking proper medical solutions. Lack of knowledge of identify the features of mental illness and ignorance about the access treatment, prejudice against people who have mental illness. This reason is most people to avoid the treatment and delays for care. Generally, the factor of poor understanding of mental illness and level of mental health knowledge associated with the mental health increasingly (Yeap \& Low, 2009).

\section{Stigma}

Stigma is a negative perception that created prejudice which leads to stereotype and discrimination among a group of people. More over from the mental illness perspective, Stigma has been identified as a significant barrier to help-seeking and care. The cultural context also important when studying element of beliefs regarding mental health. The cultural by ethnic background has brought a different definition of mental health. The concept of mental health amongst Malays defined by heredity, periodicity, congenital, brain stain, stress, conditioning and resistance (Razali, Khan, \& Hasanah, 1996). In Malay culture, vital parts in mental health including with the spiritual and religious factors.

Several respondents also noted that people with mental illness would commonly be referred to religious practitioners or shamans, rather than medical professionals. Generally, they agreed that people especially Malays have the tendency to associate mental illness with the term ' $g$ ila' (insanity) or 'sakit jiwa' (illness of the soul). Rather than a scientifically proven symptom, mental illness is commonly viewed as a supernatural phenomenon. A large part of the Malay society believe that mental illness come from spirit possession or as a social punishment (Deva, 1995). The culture itself have strong influence in Malay society as the general concept of mental illness is an outcome of abandoning or neglecting traditional values(Haque \& Masuan, 2002).

Malay population which is represent as the largest race in Malaysia often associated mental illness with the supernatural causes, God punishment, and excessive mental exertion (Khan et al., 2010) Similarly with the Malay culture, the Chinese concepts of mental health also influenced by the traditional Chinese medical beliefs based on Confucianism and Taoism (Yip, 2005) where these principles refer to the concepts of Yin and Yan as the symbol of life. Most of the Chinese believe that imbalance of the Yin-Yang can cause to the mental illness. (Yip, 2005) comparatively to the Indian believe the concept of mental health amongst Indian is centralized to the concept of the mind body dichotomy(Wig, 1999) Whereby it indicates the four aims of life "Dharma", "Kama", "Artha" and "Moksha". Indian believe that who have suffers with the mental illness problems is cause of imbalance of these four elements. As can been seen the elements of the cultural sensitivity of the different faiths and belief systems are deeply influenced by the culture and religious.

This situation may be associated with society in taking traditional way in treating mental illness. There are numerous studies that investigate about the relationship between mental health and religious belief (Campus, n.d.) The stigma itself from community and policy makers looking at mental health with low regards 


\section{Policy Insurance}

A number of respondents remarked on the ambiguous coverage of the existing healthcare policies. Historically, the insurance policies that provided by insurance company did not include mental health services until after World War II, in this prior time the insurers start to cover some of the psychiatric care in the policies. Most of the insurance company did not recognise mental illness part of critical ill that need to be cure by provided proper treatment. Recently insurance schemes do not adequately address the needs of persons that being suffering with mental illness. Standard health insurance policies do not cover for the pre-existing conditions including mental illness. This situation leads to discourage of people seeking the proper treatment as of mental illness that they are suffering as they do not want tarnish the medical records.

In the Long run, there are several positive implications towards of the inclusion of the mental health insurance coverage in the policies as the mental health benefits legislation has been found to have positive association with the increased access to care, increased diagnosis of mental health conditions. As a result it leads to the reduced of the prevalence of poor mental health and reduced of the suicide rates. (Lang, 2013). As a result, mental health insurance will facilitate medical care for mentally ill and improve their outcomes, benefitting the society in the long-term.

\section{Issues}

There are several issues on mental illness that are captured and triangulated from the interview transcripts. These include their anxiety towards the rising trend of mental illness, the widespread phenomenon of work-related stress, and the negative economic impact associated with mental illness.

\section{Rising Trend of Mental Illness}

Many of the respondents felt that there is an increasing trend of mental illness among Malaysians. Nevertheless, since its considered as a taboo, people who suffer from it are deemed hesitant to discuss it openly. Malaysians, just like other Asians, are known to have a lesser degree of openness as compared to their western counterparts. Hence, anxiety towards social desirability and negative connotations associated with mental illness are thought as the barriers for these patients to disclose their symptoms to medical professionals. The respondents believe that a typical Malaysian would perceive the risk of potential embarrassments from their social circles to outweighs the benefit derived from medical and psychological consultations. Therefore, Malaysians are thought to be highly sensitive and conservative regarding the mental health issue. The respondents relate the negative outcomes of this phenomenon into recent suicidal cases that they believed should be blamed on work-related factors.

According to the Health Ministry's 2015 National Health Morbidity Survey (NHMS) statistics, its indicated the increasing number up to 4.2 million Malaysians over 16 years old illustrated as around 30 percent of the segment were experienced some form of mental illness. In 2006, it was just showed only 11.2 per cent. This is extremely alarming as there is not enough awareness on mental illnesses. The increasing of mental health illness conditions can cause of a high direct and indirect costs that 
contribute to the impact quality of life and wellbeing affected people. The most reason that indicated of increasing number of mental health cases is related to the financial constraints, family and career problems. In terms of financial it can be the problems to manage the failing to pay bills due to the financial difficulties. Some point of views the financial constraint leads the person to isolate their self and lack of support due the inability to participate in social activities

\section{Work-related Stress}

Nowadays, the issues of mental health are increasingly prevalent in the workplace. This is unsurprising since mental health is considered as one of the contributing factors towards individual's overall health status. Poor mental health is associated with the higher absence and presenteeism rates. The contribution of job control to absence is greater for those in poor mental health (Bubonya, Cobb-Clark, \& Wooden, 2017). Moreover, the occupations itself known to be associated to the high risk of mental illness. Obviously, the working environment that related to the highly challenging are more at risk contributes to the mental illnesses compared those who feel their working environment less challenging. There is another side of this where organization should put priority into mental health issues in the workplace as an integral component of employees' wellbeing. Majority of respondents felt that mental illness can build up slowly in a subconscious manner due to prolonged stress from the workplace.

Mental health problems have an impact to the employers and businesses directly by the issues of increased absenteeism and negative impacts on the productivity and profits (Rajgopal, 2010). Mental health illness contribute to the loss of productivity (Kessler et al., 2009). Additionally, according to the World Health Organization (WHO), Mental health disorders cost the global economy \$1 trillion from the loss of productivity a year, whereby the depression being the leading cause of the mental illness. Conversely, by having Positive mental health it allows people to realize their full potential, cope with the stresses of life, work productively, and make meaningful contributions to their communities. Therefore, highlights should be given to mental health and wellness to improve individual potential and increasing work performance.

\section{Negative Economic Impact}

In general, the respondents have expressed their concerns on the negative economic impact as a consequent from the widespread mental illness suffered by Malaysians. In this regard, Malaysia is not an isolated case since mental illness are recognized as a global problem that affects the productivity and socio-economic conditions of that country. Besides, the respondents acknowledge that mental illness can directly affects business owners in terms of potential revenue lost due to absenteeism and subsequent reduction in productivity levels.

The Issues of mental health is a global issue as indicates as $12 \%$ part of global burden disease. By the year 2020 they will account for nearly 15\% of disability-adjusted life-years lost to illness. In the other hand, mental disorders affect the level productivity as it is related to the economy impact due to treatment cost. Apart of the burden to the personal itself, it can be affect to the economic itself where the labour supply losses Canada Economic burden of mental illness shows that estimated around \$51 billion per year as to cover the health care costs, loss of productivity and reductions in healthy related 
INTERNATIONAL JOURNAL OF ACADEMIC RESEARCH IN BUSINESS AND SOCIAL SCIENCES

Vol. 8, No. 12, Dec, 2018, E-ISSN: $2222-6990$ C 2018 HRMARS

quality of life (Smetanin et al., n.d.). Mental disorders imposed a huge economic burden on individuals and the society in China (Xu, Wang, Wimo, \& Qiu, 2016).

\section{CONCLUSION AND RESEARCH IMPLICATIONS}

The findings of the study provide the evidence for the key issues and challenges of mental health issues in Malaysia. Mental illness can affect to every part of a person's life, including their physical health, occupation, family and social life and relationships. The results and the experience of this study clearly stated the mental health issues in Malaysia landscape is an urgent need to further enlighten by government and society to take this as serious matters. The government should highlight the mental health issue as country agenda as increase in funds for the Health Ministry to implement and execute more anti stigma campaigns aiming to develop a positive healthy public attitude in relation

The results of this study also revealed that mental health is common and can affect anyone without age, race boundaries. Greater awareness about mental health and early diagnosis can reduce the statistic of suffering from mental health related problems. Nevertheless, there is room for improvement for the findings and methods to do future research.

\section{Acknowledgment}

The authors gratefully acknowledge the help of Universiti Teknologi MARA in providing the Research Grant Scheme Fund (Project Number: 600-IRMI/MYRA 5/3/BESTARI (038/2017) and Faculty of Business and Management for supporting the research work.

\section{References}

Ahmad, N. A., Razak, M. A. A., Naidu, B. M., Awaluddin, S. M., Chan, Y. Y., Kasim, N. M., \& Ibrahim, N. (2015). Mental health problems of adults. Institute for Public Health (Ed.), National Health and Morbidity Survey, 2, 185-189.

Boeije,H. (2002). A purposeful approach to the constant comparative method in the analysis of qualitative interviews, Quality and Quantity, vol. 36 (4), 391-409.

Brown, V. A., Harris, J. A., \& Russell, J. Y. (2010). Tackling wicked problems through the transdisciplinary imagination. Earthscan.

Bubonya, M., Cobb-Clark, D. A., \& Wooden, M. (2017). Mental health and productivity at work: Does what you do matter? Labour Economics, 46, 150-165.

Bull, F. C. L., Schipper, E. C. C., Jamrozik, K., \& Blanksby, B. A. (1997). How can and do Australian doctors promote physical activity? Preventive Medicine, 26(6), 866-873.

Chowdhury, A., Islam, I., \& Lee, D. (2013). The Great Recession, jobs and social crises: policies matter. International Journal of Social Economics, 40(3), 220-245. 
INTERNATIONAL JOURNAL OF ACADEMIC RESEARCH IN BUSINESS AND SOCIAL SCIENCES

Vol. 8, No. 12, Dec, 2018, E-ISSN: $2222-6990$ C 2018 HRMARS

Deva, M. P. (1995). Medicine in Malaysia: psychiatry. The Medical Journal of Malaysia, 50, S69-71.

Haque, A., \& Masuan, K. A. (2002). Perspective: religious psychology in Malaysia. The International Journal for the Psychology of Religion, 12(4), 277-289.

Health, I. for P. (2011). National Health and Morbidity Survey 2011 (NHMS 2011). Vol. II: NonCommunicable Disease. Ministry of Health Malaysia Kuala Lumpur.

Henderson, C., Evans-Lacko, S., \& Thornicroft, G. (2013). Mental illness stigma, help seeking, and public health programs. American Journal of Public Health, 103(5), 777-780.

Kaur, J., Cheong, S. M., Mahadir Naidu, B., Kaur, G., Manickam, M. A., Mat Noor, M., ... Rosman, A. (2014). Prevalence and correlates of depression among adolescents in Malaysia. Asia Pacific Journal of Public Health, 26(5_suppl), 53S-62S.

Kessler, R. C., Aguilar-Gaxiola, S., Alonso, J., Chatterji, S., Lee, S., Ormel, J., ... Wang, P. S. (2009). The global burden of mental disorders: an update from the WHO World Mental Health (WMH) surveys. Epidemiology and Psychiatric Sciences, 18(1), 23-33.

Khan, T. M., Sulaiman, S. A. S., Hassali, M. A., Anwar, M., Wasif, G., \& Khan, A. H. (2010). Community knowledge, attitudes, and beliefs towards depression in the state of Penang, Malaysia. Community Mental Health Journal, 46(1), 87-92.

Lang, M. (2013). The impact of mental health insurance laws on state suicide rates. Health Economics, 22(1), 73-88.

Lee, M. F., \& Lai, C. S. (2017). Exploring Learners' Mental Health Profile: A study in Universiti Tun Hussein Onn Malaysia. In IOP Conference Series: Materials Science and Engineering (Vol. 226, p. 12194). IOP Publishing.

Loo, P.-W., \& Furnham, A. (2012). Public knowledge and beliefs about depression among urban and rural Chinese in Malaysia. Asian Journal of Psychiatry, 5(3), 236-245.

Organization, W. H. (2013). Mental health: a state of well-being. 2014. Report of the WHO Department of Mental Health.

Organization, W. H. (2017). Depression and other common mental disorders: global health estimates.

Rajgopal, T. (2010). Mental well-being at the workplace. Indian Journal of Occupational and Environmental Medicine, 14(3), 63.

Razali, S. M., Khan, U. A., \& Hasanah, C. I. (1996). Belief in supernatural causes of mental illness among 
INTERNATIONAL JOURNAL OF ACADEMIC RESEARCH IN BUSINESS AND SOCIAL SCIENCES

Vol. 8, No. 12, Dec, 2018, E-ISSN: $2222-6990$ C 2018 HRMARS

Malay patients: impact on treatment. Acta Psychiatrica Scandinavica, 94(4), 229-233.

Rotenstein, L. S., Ramos, M. A., Torre, M., Segal, J. B., Peluso, M. J., Guille, C., ... Mata, D. A. (2016). Prevalence of depression, depressive symptoms, and suicidal ideation among medical students: a systematic review and meta-analysis. Jama, 316(21), 2214-2236.

Smetanin, P., Stiff, D., Briante, C., Adair, C. E., Ahmad, S., \& Khan, M. (n.d.). The life and economic impact of major mental illnesses in Canada: 2011 to 2041. Toronto: RiskAnalytica, on behalf of the Mental Health Commission of Canada; 2011.

Teoh, S. L., Chong, H. Y., Aziz, S. A., Chemi, N., Othman, A. R., Zaki, N. M., ... Chaiyakunapruk, N. (2017). The economic burden of schizophrenia in Malaysia. Neuropsychiatric Disease and Treatment, 13, 1979.

Wig, N. N. (1999). Mental health and spiritual values. A view from the East. International Review of Psychiatry, 11(2-3), 92-96.

Xu, J., Wang, J., Wimo, A., \& Qiu, C. (2016). The economic burden of mental disorders in China, 20052013: implications for health policy. BMC Psychiatry, 16(1), 137.

Yeap, R., \& Low, W. Y. (2009). Mental health knowledge, attitude and help-seeking tendency: a Malaysian context. Singapore Med J, 50(12), 1169-1176.

Yip, K. S. (2005). Family caregiving of clients with mental illness in the People's Republic of China. International Journal of Psychosocial Rehabilitation, 10(1), 35-42.

Zivin, K., Eisenberg, D., Gollust, S. E., \& Golberstein, E. (2009). Persistence of mental health problems and needs in a college student population. Journal of Affective Disorders, 117(3), 180-185. 